\title{
OSCILLATORY PROPERTY OF SOLUTIONS OF SECOND ORDER DIFFERENTIAL EQUATIONS
}

\author{
TARO YOSHIZAWA
}

(Received on July 2, 1970)

In this paper we shall discuss oscillatory property of solutions of second order differential equations by applying Liapunov's second method. Consider an equation

$$
\left(r(t) x^{\prime}\right)^{\prime}+f\left(t, x, x^{\prime}\right)=0 \quad\left({ }^{\prime}=\frac{d}{d t}\right),
$$

where $r(t)>0$ is continuous on $I=[0, \infty)$ and $f(t, x, u)$ is defined and continuous on $I \times R \times R, R=(-\infty, \infty)$. To discuss oscillatory property of solutions of $(1)$, we consider an equivalent system

$$
x^{\prime}=\frac{y}{r(t)}, \quad y^{\prime}=-f\left(t, x, \frac{y}{r(t)}\right)
$$

A solution $x(t)$ of (1) which exists in the future is said to be oscillatory if for every $T>0$ there exists a $t_{0}>T$ such that $x\left(t_{0}\right)=0$. Moreover, the equation (1) is said to be oscillatory if every solution of (1) which exists in the future is oscillatory.

THEOREM 1. Assume that there exist two continuous functions $V(t, x, y)$ and $W(t, x, y)$ which are defined on $t \geqq T, x>0,|y|<\infty$ and $t \geqq T, x<0$, $|y|<\infty$, respectively, where $T$ can be large, and assume that $V(t, x, y)$ and $W(t, x, y)$ satisfy the following conditions;

(i) $V(t, x, y) \rightarrow \infty$ uniformly for $x>0$ and $-\infty<y<\infty$ as $t \rightarrow \infty$, and $W(t, x, y) \rightarrow \infty$ uniformly for $x<0$ and $-\infty<y<\infty$ as $t \rightarrow \infty$,

(ii) $\dot{V}_{(2)}(t, x(t), y(t)) \leqq 0$ for all sufficiently large $t$, where $\{x(t), y(t)\}$ is a solution of (2) such that $x(t)>0$ for all large $t$ and

$$
\dot{V}_{(2)}(t, x(t), y(t))=\varlimsup_{h \rightarrow 0^{+}} \frac{1}{h}\{V(t+h, x(t+h), y(t+h))-V(t, x(t), y(t))\},
$$


(iii) $\dot{W}_{(2)}(t, x(t), y(t)) \leqq 0$ for all sufficiently large $t$, where $\{x(t), y(t)\}$ is a solution of $(2)$ such that $x(t)<0$ for all large $t$ and

$$
\left.\dot{W}_{(2)}(t, x(t), y(t))=\varlimsup_{h \rightarrow 0^{+}} \frac{1}{h}\{W(t+h), x(t+h), y(t+h))-W(t, x(t), y(t))\right\}
$$

Then the equation ( 1 ) is oscillatory.

ProOF. Let $x(t)$ be a solution of (1) which is defined on $\left[t_{0}, \infty\right)$, and suppose that $x(t)$ is not oscillatory. Then $x(t)$ is either positive or negative for all large $t$. Now assume that $x(t)>0$ for all $t \geqq \sigma$, where we can assume $\sigma$ to be sufficiently large. By the condition (i), if $t$ is sufficiently large, say $t \geqq t_{1}$, we have

$$
V(\sigma, x(\sigma), y(\sigma))<V(t, x, y)
$$

for all $x>0,|y|<\infty$. However, by the condition (ii), we have

$$
V(t, x(t), y(t)) \leqq V(\sigma, x(\sigma), y(\sigma)) \text { for all } t \geqq \sigma,
$$

which contradicts $V(\sigma, x(\sigma), y(\sigma))<V\left(t_{1}, x\left(t_{1}\right), y\left(t_{1}\right)\right)$. When we assume that $x(t)<0$ for all large $t$, we have also a contradiction by considering $W(t, x(t), y(t))$. Thus we see that $x(t)$ is oscillatory.

To apply this theorem, the following lemmas play an important role. In the following, a scalar function $v(t, x, y)$ will be called a Liapunov function for (2), if $v(t, x, y)$ is continuous in $(t, x, y)$ in the domain of definition and is locally Lipschitzian in $(x, y)$. Moreover, we define $\dot{v}_{(2)}(t, x, y)$ by

$$
\dot{v}_{(2)}(t, x, y)=\varlimsup_{h \rightarrow 0^{+}} \frac{1}{h}\left\{v\left(t+h, x+h \frac{y}{r(t)}, y-h f\left(t, x, \frac{y}{r(t)}\right)\right)-v(t, x, y)\right\} .
$$

If $\dot{v}_{(2)}(t, x, y) \leqq 0, v(t, x(t), y(t))$ is nonincreasing in $\mathrm{t}$, where $\{x(t), y(t)\}$ is a solution of $(2)$, see [5].

LEMMA 1. For $t \geqq T^{*}, x>0,-\infty<y<\infty$, where $T^{*}$ can be large, we assume that there exists a Liapunov function $v(t, x, y)$ which satisfies the following conditions;

(i) $y v(t, x, y)>0$ for $y \neq 0, t \geqq T^{*}, x>0$,

(ii) $\dot{v}_{(2)}(t, x, y) \leqq-\lambda(t)$, where $\lambda(t)$ is a continuous function defined on $t \geqq T^{*}$ and 


$$
\lim _{t \rightarrow \infty} \int_{T}^{t} \lambda(s) d s \geqq 0 \quad \text { for all large } T \text {. }
$$

Moreover, we assume that there exists $a \tau$ and $a w(t, x, y)$ for all large $T$ such that $\tau \geqq T$ and $w(t, x, y)$ is a Liapunov function defined on $t \geqq \tau$, $x>0, y<0$, which satisfies the following conditions;

(iii) $y \leqq w(t, x, y)$ and $w(\tau, x, y) \leqq b(y)$, where $b(y)$ is continuous, $b(0)=0$ and_b(y)<0 $(y \neq 0)$,

(iv) $\quad \dot{w}_{(2)}(t, x, y) \leqq-\rho(t) w(t, x, y)$, where $\rho(t) \geqq 0$ is continuous and

$$
\int_{\tau}^{\infty} \frac{1}{r(t)} \exp \left\{-\int_{\tau}^{t} \rho(s) d s\right\} d t=\infty .
$$

Then, if $\{x(t), y(t)\}$ is a solution of (2) such that $x(t)>0$ for all large $t$, then $y(t) \geqq 0$ for all large $t$.

PROOF. Suppose that there is a sequence $\left\{t_{n}\right\}$ such that $t_{n} \rightarrow \infty$ as $n \rightarrow \infty$ and $y\left(t_{n}\right)<0$. We can assume that $t_{n} \geqq T^{*}$ and $t_{n}$ is sufficiently large so that

$$
\lim _{t \rightarrow \infty} \int_{t_{n}}^{t} \lambda(s) d s \geqq 0, x(t)>0 \text { for } t \geqq t_{n} .
$$

Consider the function $v(t, x(t), y(t))$ for $t \geqq t_{n}$. Then we have

$$
v(t, x(t), \mathrm{y}(t)) \leqq v\left(t_{n}, x\left(t_{n}\right), y\left(t_{n}\right)\right)-\int_{t_{n}}^{t} \lambda(s) d s
$$

From (6) it follows that there is a $T_{1}>0$ such that for all $t \geqq T_{1}$,

$$
\int_{t_{n}}^{t} \lambda(s) d s \geqq \frac{1}{2} v\left(t_{n}, x\left(t_{n}\right), y\left(t_{n}\right)\right),
$$

because $v\left(t_{n}, x\left(t_{n}\right), y\left(t_{n}\right)\right)<0$. Therefore, for $t \geqq T_{1}$, we have

$$
v(t, x(t), y(t)) \leqq \frac{1}{2} v\left(t_{n}, x\left(t_{n}\right), y\left(t_{n}\right)\right)<0
$$

which implies that $y(t)<0$ for all $t \geqq T_{1}$.

For $T_{1}$, there is a $\tau$ such that $\tau \geqq T_{1}$ and there is a Liapunov function $w(t, x, y)$ defined on $t \geqq \tau, x>0, y<0$. For this $w(t, x, y)$, we have 


$$
y(t) \leqq w(t, x(t), y(t)) \leqq w(\tau, x(\tau), y(\tau)) e^{-\int_{\tau}^{t} \rho(s) d s} \leqq b(y(\tau)) e^{-\int_{\tau}^{t} \rho(s) d s}
$$

for $t \geqq \tau$. Since $x^{\prime}(t)=\frac{y(t)}{r(t)}$, we have

$$
x^{\prime}(t) \leqq b(y(\tau)) \frac{1}{r(t)} e^{-\int_{\tau}^{t} \rho(s) d s}
$$

and hence

$$
x(t) \leqq x(\tau)+b(y(\tau)) \int_{\tau}^{t} \frac{1}{r(u)} e^{-\int_{\tau}^{u} \rho(\mathbf{s}) d s} d u
$$

Since $x(t)>0$ for $t \geqq \tau$ and $b(y(\tau))<0$, there arises a contradiction by ( 5$)$. Thus we see that $y(t) \geqq 0$ for all large $t$.

REMARK. In the case where $r(t) \equiv 1$ and $\rho(t) \equiv 0$, condition (iii) can be replaced by

(iii)' $\quad a(y) \leqq w(t, x, y)$ and $w(\tau, x, y) \leqq b(y)$, where $a(y)$ is monotone, continuous, $a(0)=0, a(y)<0(y \neq 0)$ and $b(y)$ is continuous, $b(0)=0, b(y)<0(y \neq 0)$.

By the same argument, we can prove the following lemma.

LEMMA 2. For $t \geqq T^{*}, x<0,-\infty<y<\infty$, where $T^{*}$ can be large, we assume that there exists a Liapunov function $v(t, x, y)$ which satisfies the following conditions;

(i) $y v(t, x, y)<0$ for $y \neq 0, t \geqq T^{*}, x<0$,

(ii) $\dot{v}_{(2)}(t, x, y) \leqq-\lambda(t)$, where $\lambda(t)$ is a continuous function defined on $t \geqq T^{*}$ and

$$
\lim _{t \rightarrow \infty} \int_{T}^{t} \lambda(s) d s \geqq 0 \text { for all large } T \text {. }
$$

Moreover, we assume that there exists $a \tau$ and $a w(t, x, y)$ for all large $T$ such that $\boldsymbol{\tau} \geqq T$ and $w(t, x, y)$ is a Liapunov function defined on $t \geqq \tau$, $x<0, y>0$, which satisfies the following conditions;

(iii) $-y \leqq w(t, x, y)$ and $w(\tau, x, y) \leqq b(y)$, where $b(y)$ is continuous, $b(0)=0$ and $b(y)<0 \quad(y \neq 0)$,

(iv) $\dot{w}_{(2)}(t, x, y) \leqq-\rho(t) w(t, x, y)$, where $\rho(t) \geqq 0$ is continuous and 


$$
\int_{\tau}^{\infty} \frac{1}{r(t)} \exp \left\{-\int_{\tau}^{t} \rho(s) d s\right\}=\infty .
$$

Then, if $\{x(t), y(t)\}$ is a solution of (2) such that $x(t)<0$ for all large $t$, then $y(t) \leqq 0$ for all large $t$.

If we can find Liapunov functions which satisfy the conditions in Lemmas 1 and 2 , we can prove the following theorem by the same idea as in the proof of Theorem 1.

THEOREM 2. Under the assumptions of Lemmas 1 and 2, we assume that for each $\delta>0$, there exist a $T(\delta)>0$ and Liapunov functions $V(t, x, y)$ and $W(t, x, y)$ which are defined on $t \geqq T(\delta), x>\delta, y \geqq 0$ and $t \geqq T(\delta)$, $x<-\delta, y \leqq 0$, respectivel $y$, and assume that $V(t, x, y)$ and $W(t, x, y)$ satisfy the following conditions;

(i) $V(t, x, y)$ and $W(t, x, y)$ tend to infinity uniformly for $x$ and $y$ as $t \rightarrow \infty$,

(ii) $\dot{V}_{(2)}(t, x, y) \leqq 0$ as long as $\dot{V}_{(2)}$ is defined,

(iii) $\dot{W}_{(2)}(t, x, y) \leqq 0$ as long as $\dot{W}_{(2)}$ is defined.

Then the equation (1) is oscillatory.

Since we assume the existence of Liapunov functions satisfying the conditions in Lemmas 1 and 2, if $x(t)>0$ in the future, then $x(t)>\delta$ in the future for some $\delta>0$, because $x^{\prime}(t)=\frac{y(t)}{r(t)} \geqq 0$ in the future, and the similar for a solution $x(t)<0$.

EXAmPLE 1. Consider the equation (1) and assume that the following conditions are satisfied:

(a)

$$
\int_{0}^{\infty} \frac{d t}{r(t)}=\infty
$$

(b) For $t \geqq 0$ and $x \geqq 0$, there exists a continuous function $a(t)$ and an $\alpha(x)$ such that

$$
\lim _{t \rightarrow \infty} \int_{T}^{t} a(s) d s \geqq 0 \text { for all large } T
$$


and that $x \alpha(x)>0(x \neq 0), \alpha^{\prime}(x) \geqq 0$ and for all large $t, x \geqq 0,|u|<\infty$

$$
a(t) \alpha(x) \leqq f(t, x, u)
$$

(c) For $t \geqq 0$ and $x \leqq 0$, there exists a continuous function $b(t)$ and a $\beta(x)$ such that

$$
\lim _{t \rightarrow \infty} \int_{T}^{t} b(s) d s \geqq 0 \text { for all large } T
$$

and that $x \beta(x)>0(x \neq 0), \beta^{\prime}(x) \geqq 0$ and for all large $t, x \leqq 0,|u|<\infty$

$$
f(t, x, u) \leqq b(t) \beta(x)
$$

Under the assumptions above, if $\{x(t), y(t)\}$ is a solution of (2) such that $x(t)>0$ for all large $t$, then $y(t) \geqq 0$ for all large $t$. To see this, we can assume that (9) through (12) hold good for all $t \geqq T^{*}$ and all $T \geqq T^{*}$. For $t \geqq T^{*}$, $x>0,|y|<\infty$, define $v(t, x, y)$ by

$$
v(t, x, y)=\frac{y}{\alpha(x)}
$$

Then, we have

$$
\dot{\bar{v}}_{(2)}(t, x, y)=\frac{1}{\alpha^{2}(x)}\left\{-f\left(t, x, \frac{y}{r(t)}\right) \alpha(x)-y \alpha^{\prime}(x) \frac{y}{r(t)}\right\} \leqq-a(t) .
$$

Hence this $v(t, x, y)$ satisfies the conditions in Lemma 1 with $\lambda(t)=a(t)$.

Since the condition ( 9 ) implies that for all $T \geqq T^{*}$, there is a $\tau$ such that $\tau \geqq T$ and

$$
\int_{\tau}^{t} a(s) d s \geqq 0 \text { for all } t \geqq \tau,
$$

a function $w(t, x, y)=y+\alpha(x) \int_{\tau}^{t} a(s) d s$ defined on $t \geqq \tau, x>0, y<0$ satisfies the conditions in Lemma 1 with $\rho(t) \equiv 0$. Thus the conclusion follows from Lemma 1 . If we consider functions

$$
v(t, x, y)=\frac{y}{\beta(x)}, t \geqq T^{*}, x<0,|y|<\infty,
$$




$$
w(t, x, y)=-y-\beta(x) \int_{\tau}^{t} b(s) d s, \quad t \geqq \tau, x<0, y>0,
$$

from Lemma 2 it follows that if $\{x(t), y(t)\}$ is a solution of (2) such that $x(t)<0$ for all large $t$, then $y(t) \leqq 0$ for all large $t$.

Under the assumptions (a), (b) and (c), we shall discuss oscillatory property of solutions of (1). The following results contain Macki and Wong's result [3], Coles' result [2] and others.

( I) If we have

$$
\int_{0}^{\infty} a(s) d s=\infty, \quad \int_{0}^{\infty} b(s) d s=\infty,
$$

then the equation $(1)$ is oscillatory.

For $t \geqq T^{*}, x>0$ and $-\infty<y<\infty$, set

$$
V(t, x, y)= \begin{cases}\frac{y}{\alpha(x)}+\int_{0}^{t} a(s) d s & (y \geqq 0) \\ \int_{0}^{t} a(s) d s & (y<0) .\end{cases}
$$

Then, clearly $V(t, x, y) \rightarrow \infty$ uniformly for $x>0$ and $-\infty<y<\infty$, and we have

$$
\begin{aligned}
\dot{V}_{(2)}(t, x, y) & =\frac{1}{\alpha^{2}(x)}\left\{-f\left(t, x, \frac{y}{r(t)}\right) \alpha(x)-y \alpha^{\prime}(x) \frac{y}{r(t)}\right\}+a(t) \\
& \leqq-a(t)+a(t) \\
& \leqq 0
\end{aligned}
$$

for $t \geqq T^{*}, x>0$ and $y \geqq 0$. Therefore, $V(t, x, y)$ satisfies the conditions in Theorem 1. Similarly,

$$
W(t, x, y)= \begin{cases}\int_{0}^{t} b(s) d s & (y>0) \\ \frac{y}{\beta(x)}+\int_{0}^{t} b(s) d s & (y \leqq 0)\end{cases}
$$


satisfies the conditions in Theorem 1. Thus the conclusion follows from Theorem 1.

(II) If we have

$$
\begin{aligned}
& \int_{0}^{\infty} a(s) d s<\infty, \int_{0}^{t}\left(\frac{1}{r(s)} \int_{s}^{\infty} a(u) d u\right) d s \rightarrow \infty \text { as } t \rightarrow \infty, \\
& \int_{0}^{\infty} b(s) d s<\infty, \int_{0}^{t}\left(\frac{1}{r(s)} \int_{s}^{\infty} b(u) d u\right) d s \rightarrow \infty \text { as } t \rightarrow \infty,
\end{aligned}
$$

$$
\int_{\varepsilon}^{\infty} \frac{d u}{\alpha(u)}<\infty \text { for some } \varepsilon>0
$$

and

$$
\int_{-\varepsilon}^{-\infty} \frac{d u}{\beta(u)}<\infty \text { for some } \varepsilon>0
$$

the equation ( 1 ) is ocillatory.

For $t \geqq T^{*}, x>0,|y|<\infty$, set

$$
V(t, x, y)=\int_{x}^{\infty} \frac{d u}{\alpha(u)}+\int_{0}^{t}\left(\frac{1}{r(s)} \int_{s}^{\infty} a(u) d u\right) d s
$$

For a solution $x(t)$ which satisfies, $x(t)>0$ for all large $t$, we can assume that $x(t)>0, y(t) \geqq 0$ for $t \geqq \sigma, \sigma$ sufficiently large, and hence

$$
\begin{aligned}
\dot{V}(t, x(t), y(t)) & =-\frac{1}{\alpha(x(t))} \frac{y(t)}{r(t)}+\frac{1}{r(t)} \int_{t}^{\infty} a(u) d u \\
& =\frac{1}{r(t)}\left\{-\frac{y(t)}{\alpha(x(t))}+\int_{t}^{\infty} a(u) d u\right\} .
\end{aligned}
$$

If we set $V^{*}(t, x, y)=-\frac{y}{\alpha(x)}+\int_{t}^{\infty} a(u) d u, V^{*}(t, x(t), y(t)) \leqq \int_{t}^{\infty} a(u) d u$, and hence

$$
\varlimsup_{t \rightarrow \infty} V^{*}(t, x(t), y(t)) \leqq 0
$$


On the other hand, we have

$$
\begin{aligned}
\dot{V}_{(2)}^{*}(t, x, y) & =-\frac{1}{\alpha^{2}(x)}\left\{-f\left(t, x, \frac{y}{r(t)}\right) \alpha(x)-y \alpha^{\prime}(x) \frac{y}{r(t)}\right\}-a(t) \\
& \geqq 0 .
\end{aligned}
$$

Therefore $V^{*}(t, x(t), y(t)) \leqq 0$ and consequently $\dot{V}(t, x(t), y(t)) \leqq 0$ for $t \geqq \sigma$.

Similarly, if we define $W(t, x, y)$ by

$$
W(t, x, y)=\int_{x}^{-\infty} \frac{d u}{\beta(u)}+\int_{0}^{t}\left(\frac{1}{r(s)} \int_{s}^{\infty} b(u) d u\right) d s,
$$

this $W(t, x, y)$ satisfies the conditions in Theorem 1 . Thus the conclusion follows from Theorem 1 .

REMARK 1. It is clear that we can combine the conditions in (I) and (II). For example, if

$$
\int_{0}^{\infty} a(s) d s=\infty, \int_{0}^{t}\left(\frac{1}{r(s)} \int_{s}^{\infty} b(u) d u\right) d s \rightarrow \infty \text { as } t \rightarrow \infty
$$

and

$$
\int_{-\varepsilon}^{-\infty} \frac{d u}{\beta(u)}<\infty \text { for some } \varepsilon>0
$$

then the equation ( 1 ) is oscillatory.

REMARK 2. If a continuous function $a(t)$ satisfies (9), then $\int_{0}^{\infty} a(s) d s=\infty$ or $\int_{0}^{\infty} a(s) d s$ exists. Macki and Wong assumed $\alpha(x)$ and $\beta(x)$ to be nondecreasing, but we can find an $\alpha(x)$ and a $\beta(x)$ which have their derivatives, because $a(t)$, $b(t)$ are nonnegative in their case.

(III) If there exist a constant $m>0$ and two positive differentiable functions $h(t)$ and $g(t)$ defined on $I$ such that $\alpha^{\prime}(x) \geqq m, \beta^{\prime}(x) \geqq m$ and

$$
\int_{0}^{t} h(s)\left\{a(s)-\frac{1}{4} \frac{r(s)}{m}\left(\frac{h^{\prime}(s)}{h(s)}\right)^{2}\right\} d s \rightarrow \infty \text { as } t \rightarrow \infty,
$$




$$
\int_{0}^{t} g(s)\left\{b(s)-\frac{1}{4} \frac{r(s)}{m}\left(\frac{g^{\prime}(s)}{g(s)}\right)^{2}\right\} d s \rightarrow \infty \text { as } t \rightarrow \infty,
$$

the equation ( 1 ) is oscillatory.

This is a generalization of a result of Opial [4], and in this case,

$$
\begin{aligned}
V(t, x, y) & =\frac{y}{\alpha(x)} h(t)+\int_{0}^{t} h(s)\left\{a(s)-\frac{1}{4} \frac{r(s)}{m}\left(\frac{h^{\prime}(s)}{h(s)}\right)^{2}\right\} d s(x>0, y \geqq 0), \\
W(t, x, y) & =\frac{y}{\beta(x)} g(t)+\int_{0}^{t} g(s)\left\{b(s)-\frac{1}{4} \frac{r(s)}{m}\left(\frac{g^{\prime}(s)}{g(s)}\right)^{2}\right\} d s(x<0, y \leqq 0)
\end{aligned}
$$

satisfy the conditions in Theorem 1 .

LEMMA 3. In addition to the assumption of Lemma 1, assume that there exists a Liapunov function $u(t, x, y)$ defined on $t \geqq T^{*}, x>0, y>R(R>0$ : large), which satisfies

(i) $u(t, x, y) \rightarrow \infty$ uniformly for $t, x$ as $y \rightarrow \infty$, and $u(t, x, y) \leqq \gamma(y)$, where $\gamma(r)>0$ is continuous,

(ii) $\quad \dot{u}_{(2)}(t, x, y) \leqq 0$.

Then, if $\{x(t), y(t)\}$ is a solution of (2) such that $x(\mathrm{t})>0$ for all large $t$, then $y(t)$ is bounded for all large $t$.

PROOF. Let $x(t)>0$ and $y(t) \geqq 0$ for $t \geqq \sigma, \sigma \geqq T^{*}$. By Lemma 1 , there is such a $\sigma$. Let $\mathrm{k}$ be such that $y(\sigma)<K, K>R$. There is a constant $\gamma^{*}>0$ such that $u(t, x, K) \leqq \gamma^{*}$, and there also exists an $M>0$ for which we have $\gamma^{*}<u(t, x, M)$ for all $t \geqq \sigma$ and $x>0$ by the condition (i). But there arises a contradiction by (ii), which shows that $0 \leqq y(t)<M$ for all $t \geqq \sigma$.

LEMMA 4. In addition to the assumption of Lemma 2, assume that there exists a Liapunov function $u(t, x, y)$ defined on $t \geqq T^{*}, x<0, y<-R(R>0$ : large), which satisfies

(i) $u(t, x, y) \rightarrow \infty$ uniformly for $t, x$ as $y \rightarrow-\infty$, and $u(t, x, y) \leqq \gamma(|y|)$, where $\gamma(r)>0$ is continuous,

(ii) $\dot{u}_{(2)}(t, x, y) \leqq 0$.

Then, if $\{x(t), y(t)\}$ is a solution of (2) such that $x(t)<0$ for all large $t$, then $y(t)$ is bounded for all large $t$. 
THEOREM 3. Under the assumptions of Lemmas 3 and 4, we assume that for each $\delta>0$ and $m>0$, there exists a $T(\delta, m)>0$ and two Liapunov functions $V(t, x, y)$ and $W(t, x, y)$ such that $V(t, x, y)$ is defined on $t \geqq$ $T(\delta, m), x>\delta, 0 \leqq y<m$ and $W(t, x, y)$ is defined on $t \geqq T(\delta, m), \quad x<-\delta$, $-m<y \leqq 0$, and we assume that $V(t, x, y)$ and $W(t, x, y)$ satisfy the following conditions;

(i) $V(t, x, y)$ and $W(t, x, y)$ tend to infinity uniformly for $x, y$ as $t \rightarrow \infty$,

(ii) $\dot{V}_{(2)}(t, x, y) \leqq 0$ as long as $\dot{V}_{(2)}$ is defined,

(iii) $\dot{W}_{(2)}(t, x, y) \leqq 0$ as long as $\dot{W}_{(2)}$ is defined.

Then the equation (1) is oscillatory.

PROOF. Let $x(t)$ be a solution of (1) which exists in the future, and suppose that $x(t)$ is not oscillatory. Then $x(t)$ is either positive or negative for all large $t$. Now assume that $x(t)>0$ for all large $t$. By Lemma 1, we can see that there is a $t_{1}>0$ such that $x(t)>0, y(t) \geqq 0$ for all $t \geqq t_{1}$, where we can assume that $t_{1} \geqq T^{*}$. By Lemma 3 , there is an $m>0$ such that $0 \leqq y(t)<m$ for all $t \geqq t_{1}$. Since $x^{\prime}(t)=\frac{y(t)}{r(t)} \geqq 0$ for $t \geqq t_{1}$, we have $x(t) \geqq x\left(t_{1}\right)>0$ for $t \geqq t_{1}$. Consider the Liapunov function $V(t, x, y)$ defined for $t \geqq T(\delta, m), x>\delta, 0 \leqq y<m$, where $\delta=x\left(t_{1}\right) / 2$ and we can assume $T \geqq t_{1}$. Then, by the same argument as in the proof of Theorem 1 , there arises a contradiction. When $x(t)<0$ for all large $t$, we have also a contradiction by using Lemma 4 and $W(t, x, y)$. Thus we can see that the equation ( 1 ) is oscillatory.

EXAMPLE 2. (Bobisud [1]). Consider an equation

$$
x^{\prime \prime}+a\left(t, x, x^{\prime}\right) \cdot x^{\prime}+f\left(t, x, x^{\prime}\right)=0
$$

and an equivalent system

$$
x^{\prime}=y, \quad y^{\prime}=-a(t, x, y) y-f(t, x, y) .
$$

The following assumptions will be made;

(i) $f(t, x, y)$ is continuous on $I \times R \times R$ and $x f(t, x, y)>0$ for $x \neq 0$,

(ii) $a(t, x, y)$ is continuous on $I \times R \times R$ and there exist continuous nonnegative functions $k(t)$ and $p(t)$ such that

$$
-k(t) \leqq a(t, x, y) \leqq p(t) \text { for } t \in I, x \in R, y \in R,
$$


(iii) for any $\delta>0$ and $m>0$, there exists a $T(\delta, m)$ and a $g(t ; \delta, m) \geqq 0$ defined for $t \geqq T(\delta, m)$ such that

$$
\int_{T(\delta, m)}^{t} g(s ; \delta, m) d s \rightarrow \infty \text { as } t \rightarrow \infty
$$

and that $|x| \geqq \delta,|y| \leqq m$ and $x y \geqq 0$ imply $|f(t, x, y)| \geqq g(t ; \delta, m)$,

(iv)

$$
\int_{0}^{\infty} k(s) d s<\infty, \lim _{t \rightarrow \infty} \int_{0}^{t} e^{-\int_{0}^{s} p(\sigma) d \sigma} d s=\infty
$$

Then the equation (19) is oscillatory.

For this equation, it is not difficult to find Liapunov functions which satisfy the conditions in Theorem 3. For $t \geqq 0, x>0,|y|<\infty$, the function

$$
v(t, x, y)= \begin{cases}e^{-\int_{0}^{t} k(s) d s} y & (y \geqq 0) \\ e^{\int_{0}^{t} p(s) d s} y & (y<0)\end{cases}
$$

satisfies the conditions in Lemma 1 with $\lambda(t) \equiv 0$. For any $\tau \geqq 0$, the function $w(t, x, y)=y$ defined for $t \geqq \tau, x>0, y<0$ satisfies the conditions in Lemma 1 , since

$$
\begin{aligned}
\dot{w}_{(20)}(t, x, y) & =-a(t, x, y) y-f(t, x, y) \\
& \leqq-p(t) y \\
& \leqq-p(t) w(t, x, y)
\end{aligned}
$$

and

$$
\int_{\tau}^{\infty} e^{-\int_{\tau}^{t} p(s) d s} d t=\infty
$$

Moreover, it is easily seen that $u(t, x, y)=y^{2} \exp \left(-2 \int_{0}^{t} k(s) d s\right)$ satisfies the conditions in Lemma 3 , since $\int_{0}^{\infty} k(t) d t<\infty$ and $f(t, x, y)>0$. Furthermore, we can see that 


$$
\begin{gathered}
v(t, x, y)=\left\{\begin{array}{cc}
e^{\int_{0}^{t} p(s) d s}(-y) & (t \geqq 0, x<0, y \geqq 0) \\
e^{-\int_{0}^{t} k(s) d s}(-y) & (t \geqq 0, x<0, y<0),
\end{array}\right. \\
w(t, x, y)=-y \quad(t \geqq \tau, x<0, y>0)
\end{gathered}
$$

and

$$
u(t, x, y)=e^{-2 \int_{0}^{t} k(s) d s} y^{2} \quad(t \geqq 0, x<0, y<0)
$$

satisfy the conditions in Lemma 4. Next, for each $\delta>0$ and $m>0$, define $V(t, x, y)$ for $t \geqq T(\delta, m), x>\delta, 0 \leqq y<m$ by

$$
V(t, x, y)=e^{-\int_{0}^{t} k(s) d s} y+L \int_{T(\delta, m)}^{t} g(s ; \delta, m) d s,
$$

where $L=e^{-\int_{0}^{\infty} k(s) d s}>0$. Then we have

$$
\begin{aligned}
\dot{V}_{(20)}(t, x, y) & =e^{-\int_{0}^{t} k(s) d s}\{-k(t) y-a(t, x, y) y-f(t, x, y)\}+L g(t ; \delta, m) \\
& \leqq e^{-\int_{0}^{t} k(s) d s}\{-k(t) y+k(t) y-g(t ; \delta, m)\}+L g(t ; \delta, m) \\
& \leqq-e^{-\int_{0}^{t} k(s) d s} g(t ; \delta, m)+L g(t ; \delta, m) \leqq 0
\end{aligned}
$$

Thus we see that $V(t, x, y)$ satisfies the conditions in Theorem 3. Similarly,

$$
W(t, x, y)=e^{-\int_{0}^{t} k(s) d s}(-y)+L \int_{T(\delta, m)}^{t} g(s ; \delta, m) d s
$$

is the desired one. Thus it follows from Theorem 3 that the equation (19) is oscillatory.

EXAMPLE 3. For the equation (19), we assume ( $i$ ) and (ii), and instead of (iii), (iv), we assume that

(iii) $^{\prime}$ for any $\delta>0$, there exists a $T(\delta)>0$ and a $g(t ; \delta) \geqq 0$ defined for $t \geqq T(\delta)$ such that

$$
e^{-\int_{0}^{t} k(s) d s} \int_{T(\delta)}^{t} g(s ; \delta) d s \rightarrow \infty \text { as } t \rightarrow \infty
$$


and that $|x| \geqq \delta, x y \geqq 0$ imply $|f(t, x, y)| \geqq g(t ; \delta)$,

(iv)'

$$
\lim _{t \rightarrow \infty} \int_{0}^{t} e^{-\int_{0}^{s} p(\sigma) d \sigma} d s=\infty
$$

Then the equation (19) is oscillatory.

From conditions ( i ), (ii) and (iv)', it follows that there are Liapunov functions which satisfy the conditions in Lemmas 1 and 2, as was seen in Example 2. For $t \geqq T(\delta), x>\delta, y \geqq 0$, define

$$
V(t, x, y)=e^{-\int_{0}^{t} k(s) d s} y+e^{-\int_{0} t k(s) d s} \int_{T(\delta)}^{t} g(s ; \delta) d s .
$$

Then we have

$$
\begin{aligned}
\dot{V}_{(20)}(t, x, y)= & e^{-\int_{0}^{t} k(s) d s}\{-k(t) y-a(t, x, y) y-f(t, x, y)\} \\
& +e^{-\int_{0}^{t} k(s) d s}\left\{-k(t) \int_{T(\delta)}^{t} g(s ; \delta) d s+g(t ; \delta)\right\} \\
\leqq & e^{-\int_{0}^{t} k(s) d s}\{-k(t) y+k(t) y-g(t ; \delta)\} \\
& -k(t) e^{-\int_{0}^{t} k(s) d s} \int_{T(\delta)}^{t} g(s ; \delta) d s+e^{-\int_{0}^{t} k(s) d s} g(t ; \delta)
\end{aligned}
$$

$\leqq 0$

For $t \geqq T(\delta), x<-\delta, y \leqq 0$, if we define $W(t, x, y)$ by

$$
W(t, x, y)=e^{-\int_{0}^{t} k(s) d s}(-y)+e^{-\int_{0}^{t} k(s) d s} \int_{T(\delta)}^{t} g(s ; \delta) d s,
$$

we have also $\dot{W}_{(20)}(t, x, y) \leqq 0$. Therefore we can conclude by Theorem 2 that the equation (19) is oscillatory.

REMARK. For the equation (19), Bobisud claimed in [1] that the equation (19) is oscillatory under the assumptions ( i), (ii) in Example 2 and

(iii)" given $\delta>0$ there exists a $T(\delta)>0$ and a $g(t ; \delta) \geqq 0$ defind fọr 
$t \geqq T(\delta)$ with

$$
\frac{1}{t} \int_{T(\delta)}^{t}(t-s) g(s ; \delta) d s \rightarrow \infty \text { as } t \rightarrow \infty
$$

and such that $|x| \geqq \delta, x y>0$ imply $|f(t, x, y)| \geqq g(t ; \delta)$,

(iv)" for any $t_{1}, t_{2}>0$,

$$
\frac{1}{t} \int_{t_{1}}^{t} k(\sigma) e^{\left.\int_{t_{2}}^{\sigma} k i \boldsymbol{s}\right) d s} d \sigma
$$

is bounded from above and

$$
\lim _{t \rightarrow \infty} \int_{t_{1}}^{t} e^{-\int_{t_{1}}^{s} p(\sigma) d \sigma} d s=\infty
$$

However, there is a mistake in his proof, and actually his result is not necessarily true as the following example shows. Consider an equation

$$
x^{\prime \prime}-\frac{x}{t+1}+f(t, x)=0
$$

where

$$
f(t, x)=\left\{\begin{array}{lc}
\frac{1}{t+1} & (x \geqq 1) \\
\frac{x}{t+1} & (|x|<1) \\
-\frac{1}{t+1} & (x \leqq-1) .
\end{array}\right.
$$

This equation satisfies the conditions above, but it has solutions $x=t+1$ and $x=-t-1$ which are not oscillatory.

Under the condition (iv)", which is equivalent to

(iv) $)^{\prime \prime \prime}$ for some $t_{0}>0$

$$
\frac{1}{t} e^{\int_{t_{0}}^{t_{k}(s) d s}}<M \quad \text { for } t \geqq t_{0}
$$

and 


$$
\lim _{t \rightarrow \infty} \int_{0}^{t} e^{-\int_{0}^{s} p(\sigma) d \sigma} d s=\infty
$$

if we assume

$$
\frac{1}{t} \int_{T(\delta)}^{t} g(s ; \delta) d s \rightarrow \infty \text { as } t \rightarrow \infty
$$

in place of (22), the equation (19) is oscillatory, because (24) and (25) imply (21).

\section{REFERENCES}

[1] L. E. BoBISUD, Oscillation of nonlinear differential equations with small nonlinear damping, SIAM J. Appl. Math., 18(1970), 74-76.

[2] W. J. Coles, Oscillation criteria for nonlinear second order equations, Ann. Mat. Pura Appl., 82(1969), 123-133.

[ 3 ] J.W. MACKI AND J.S. W. WONG, Oscillation of solutions to second-order nonlinear differential equations, Pacıfic J. Math., 24(1968), 111-117.

[4] Z. OPIAL, Sur une critère d'oscillation des integrales de l'equation différentielle $\left(Q(t) x^{\prime}\right)^{\prime}$ $+f(t) x=0$, Ann. Polon. Math., 6(1959), 99-104.

[5] T. YoshizawA, "Stability Theory by Liapunov's Second Method." The Mathematical Society of Japan, Tokyo, 1966.

\section{MATHEMATICAL INSTITUTE}

TÔHOKU UNIVERSITY

SENDAI, JAPAN 